\title{
On the isotopic fractionation of terrestrial xenon by gravitational separation inside porous planetesimals with size distribution
}

\author{
KeIJI OHTSUKI, ${ }^{1 *}$ AKIKO TAKAHASHI ${ }^{2 \dagger}$ and MitsUyOSHI SANO ${ }^{2 \ddagger}$ \\ ${ }^{1}$ Laboratory for Atmospheric and Space Physics, University of Colorado, 392 UCB, Boulder, Colorado 80309-0392, U.S.A. \\ ${ }^{2}$ Department of Earth and Environmental Sciences, Yamagata University, Yamagata 990-8560, Japan
}

(Received November 21, 2003; Accepted April 6, 2004)

\begin{abstract}
Taking account of the size distribution of planetesimals inferred from recent theoretical studies of planetesimal accumulation, we examine gravitational separation of noble gases inside large porous planetesimals in the solar nebula. The size distribution is characterized by the value of the minimum planetesimal size $R_{0}$ for a given power-law distribution, which also determines the maximum planetesimal size for a given total mass of planetesimals. Both enrichment and isotopic fractionation of noble gases strongly depend on the value of $R_{0}$, as it determines the number of large planetesimals inside which gravitational separation of noble gases is significant. We find the ranges of the values of $R_{0}$ that can produce the enrichment and the isotopic fractionation of Xe similar to the terrestrial values separately, but the two ranges are found not to overlap. We also find significant isotopic fractionation of $\mathrm{Kr}$ for the parameter value that can produce the terrestrial isotopic fractionation of $\mathrm{Xe}$, in contrast to the observation. These results suggest that the isotopic fractionation of terrestrial Xe cannot be explained if we only consider the gravitational separation inside porous planetesimals with the size distribution.
\end{abstract}

Keywords: atmosphere, composition, planetary formation, planetesimals, origin, solar system, terrestrial planets

\section{INTRODUCTION}

Elemental and isotopic abundances of noble gases have been measured for various materials, including the Earth's atmosphere and various mantle-derived materials, meteorites, lunar samples, and the atmosphere of Mars and Venus. The similarities and differences among these observational data provide important constraints on the formation and evolution of the terrestrial planets and their atmospheres (e.g., Ozima and Podosek, 1983, 2002). However, there is still no satisfactory theory on the origin of terrestrial noble gases. In particular, there has been a long-standing interest in the elemental and isotopic composition of terrestrial Xe. The relative abundance of Xe in atmospheric noble gas is considerably lower than that in the noble gas component in meteorites. The relative deficiency of Xe in the Earth has been regarded to reflect some characteristic event during Earth's evolution, and is called "the missing xenon paradox" (e.g., Pepin, 1991; Tolstikhin and O'Nions, 1994; Ozima and Podosek, 2002). On the other hand, observational data show that the Xe isotopic patterns of the Earth's atmosphere and mantle-

\footnotetext{
*Corresponding author (e-mail: ohtsuki@lasp.colorado.edu) Graduated in March, 1999.

${ }^{\ddagger}$ Graduated in March, 2000.
}

Copyright (C) 2004 by The Geochemical Society of Japan. derived materials are practically identical, and heavier isotopes are enriched compared to the isotopic pattern of solar wind or meteorites. However, such distinct fractionation is not seen in the isotopic pattern of $\mathrm{Kr}$ (e.g., Ozima and Podosek, 2002). If we assume that the terrestrial atmosphere was derived from the primordial atmosphere with solar composition by some mechanism of mass fractionation such as hydrodynamic escape (e.g., Hunten et al., 1987), we would expect that Xe should be less depleted and less fractionated than lighter noble gases, such as $\mathrm{Kr}$. Therefore, we have to explain why Xe is more depleted and isotopically fractionated, despite being heavier than $\mathrm{Kr}$.

As a possible mechanism for creating the above noble gas patterns, Ozima and Nakazawa (1980) proposed fractionation caused by gravitational separation of solar nebula gases captured inside large porous planetesimals. In this model, the enhancement in the gas concentration increases exponentially with the atomic weight, thus isotopic fractionation becomes larger in heavier noble gas elements. When an isothermal nebula gas surrounds a constant porosity planetesimal of density $\rho_{s}$ and radius $R$, the enrichment factor $\alpha_{i}$ of a noble gas species $i$ with atomic mass $\mu_{i}$ due to the gravitational separation can be written as (Ozima and Nakazawa, 1980)

$$
\alpha_{i} \equiv 3 \exp \left(A \mu_{i}\right) \int_{0}^{1} x^{2} \exp \left(-A \mu_{i} x^{2}\right) d x
$$




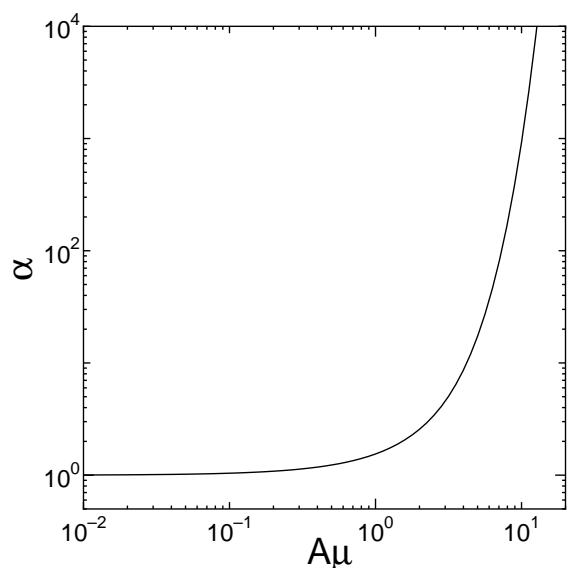

Fig. 1. Enrichment factor $\alpha$ defined by Eq. (1) is shown as a function of $A \mu$.

where $x=r / R$ is the distance from the center of the planetesimal scaled by $R$. The parameter $A$ is defined by

$$
A \equiv \frac{2 \pi G m_{\mathrm{H}} \rho_{\mathrm{s}} R^{2}}{3 k T},
$$

where $m_{\mathrm{H}}$ is the mass of a hydrogen atom, $k$ Boltzmann's constant, and $T$ the temperature. Equation (1) shows that the enrichment becomes significant when $A \mu_{i} \gtrsim 1$ (see Fig. 1). This condition can be rewritten as $R \geqslant R_{\text {crit }}$, where

$$
R_{\text {crit }} \equiv\left(\frac{3 k T}{2 \pi G m_{\mathrm{H}} \rho_{\mathrm{s}} \mu_{i}}\right)^{1 / 2}
$$

is the critical radius derived by equating the pressure gradient of the gas species $i\left(\sim k T \rho_{i} /\left(\mu_{i} m_{\mathrm{H}} R\right)\right)$ with the gravitational attraction by the planetesimal $\left(\sim \pi G \rho_{i} \rho_{s} R\right)$. For example, the values of $R_{\text {crit }}$ for $\mathrm{Ne}, \mathrm{Ar}, \mathrm{Kr}$, and $\mathrm{Xe}$ are $580 \mathrm{~km}, 430 \mathrm{~km}, 280 \mathrm{~km}$, and $230 \mathrm{~km}$, respectively, where we assumed that $T=225 \mathrm{~K}$ and $\rho_{s}=2 \mathrm{~g} \mathrm{~cm}^{-3}$ following Ozima and Nakazawa (1980). This is consistent with the results of Ozima and Nakazawa (1980; see their table 1), and they showed that the observed isotopic fractionation of terrestrial Xe could be explained by gravitational separation inside planetesimals of $R \simeq 600 \mathrm{~km}$. However, in their model, $\mathrm{Kr}$ also showed non-negligible isotopic fractionation, in contrast to the observation.

In order to solve this problem, Igarashi and Ozima (1988) took account of a size distribution of planetesimals. On the basis of the results of the numerical simulation of planetesimal accumulation by Nakagawa et al. (1983), they assumed that the size distribution of planetesimals can be approximated by

$$
N(R) \simeq\left(R / R_{0}\right) \exp \left(-R / R_{0}\right) \quad\left(0 \leq R \leq R_{\max }\right),
$$

where $R_{0}$ is the radius at the maximum of $N(R)$. Fixing the values of $R_{0}$ to be in the range of $20-30 \mathrm{~km}\left(R_{0}=30\right.$ $\mathrm{km}$ in typical cases), Igarashi and Ozima (1988) calculated the isotopic fractionation of noble gases for several values of $R_{\max }$. When $R_{\max } \simeq 10^{3} \mathrm{~km}$, they were able to approximately reproduce the observed fractionation of terrestrial $\mathrm{Xe}$, while leaving $\mathrm{Kr}$ and other lighter noble gases almost unfractionated. Igarashi and Ozima (1988) assumed the value of $R_{0}$ to be much smaller than $R_{\text {crit }}$ given by Eq. (3). As a result, the enrichment of Xe by the gravitational separation was caused mainly by a small number of large bodies near the high-mass end of the size distribution given by Eq. (4). On the other hand, they found that the total amount of heavy noble gases (i.e., Ar, Kr, and Xe) that could have been delivered to Earth in their model was too small (by two to three orders of magnitude) to account for terrestrial values. This was partly because the number of large bodies that can contribute to the noble gas enrichment (i.e., $R>R_{\text {crit }}$ ) was too small when the size distribution in the form of Eq. (4) with $R_{0} \simeq 20-30 \mathrm{~km}$ is assumed. In fact, Takahashi (1999) studied the Xe enrichment by the gravitational separation for a wide range of values of $R_{0}$ and $R_{\max }$ for the size distribution given by Eq. (4), and found that the enrichment factor can become comparable to or larger than the terrestrial values when $R_{0} \geqslant 200 \mathrm{~km}$ and $R_{\text {max }} \geqslant 850 \mathrm{~km}$. However, since both the enrichment and isotopic fractionation sensitively depend on the choice of $R_{\max }$, she found that $R_{\max }$ should be restricted in an unrealistically narrow range near $R_{\max } \sim 850 \mathrm{~km}$ to account for the terrestrial values.

As an alternative improvement of the model of Ozima and Nakazawa (1980), Zahnle et al. (1990a) took account of trapping of gravitationally fractionated gas at the critical pore-closing depth by lithostatic pressure. In this case, the isotopic fractionation is determined by the value of the critical pore-closing pressure that is a material property, thus Xe fractionation is roughly the same for all planetesimals large enough to close pores. On the basis of the data for sand and the lunar highland crust, Zahnle et al. (1990a) assumed that the critical pore-closing pressure to be 1-2 kbars, and found that the predicted degree of fractionation agrees with the terrestrial value. However, the total amount of Xe that could have been delivered to Earth in these planetesimals was more than an order of magnitude too small, unless rather efficient adsorption of Xe in small bodies and its release inside large planetesimals was assumed.

The size distribution (4) used by Igarashi and Ozima (1988) and Takahashi (1999) was based on the numerical simulation by Nakagawa et al. (1983). In this simulation, Nakagawa et al. found that a swarm of planetesimals ini- 
tially of equal size would evolve such that the large bodies were always of nearly equal size (corresponding to $R_{0}$ in Eq. (4)) and that these large bodies contained most of the mass of the swarm. Such a mode of planetary growth is called "orderly growth." On the other hand, later simulations by Wetherill and Stewart $(1989,1993)$ included the effect of equipartition of kinetic energy caused by gravitational scattering between planetesimals with different masses (which is called "dynamical friction"), which was neglected in Nakagawa et al.'s simulation. Wetherill and Stewart demonstrated that inclusion of this effect led to an alternative form of planetesimal growth termed "runaway growth," in which a small number of large bodies grow much more rapidly than the remaining smaller bodies. The size distribution of the small bodies that contain most of the mass of the swarm in this stage can be approximately given by

$$
n(m) d m \propto m^{-p} d m \text { with } p \simeq 8 / 3 \quad\left(m_{0} \leq m \leq m_{\max }\right),
$$

or, equivalently,

$$
N(R) d R \propto R^{-q} d R \text { with } q \simeq 6 \quad\left(R_{0} \leq R \leq R_{\max }\right),
$$

where $m_{0}$ and $R_{0}$ are the initial values of the mass and radius of planetesimals before accretion, and $m_{\max }$ and $R_{\max }$ are those for the largest bodies in the planetesimal swarm. These results are also confirmed by $\mathrm{N}$-body simulations (see a recent review by Kortenkamp et al., 2000). When fragmentation of planetesimals is taken into account, a number of bodies smaller than the initial size is produced. These small fragments have a shallower size distribution than the above size distribution of planetesimals formed by accretion (Eqs. (5) and (6)), and they could significantly contribute to the growth of runaway bodies in the late stage of accretion (Wetherill and Stewart, 1993; Ohtsuki et al., 2002). However, we neglect those small fragments in the present study, since the amount of noble gases captured inside planetesimals is significant only for large bodies.

In the present work, using the size distribution given by Eq. (5), which is inferred from recent theoretical studies of planetary accretion, we re-consider the problem of the Xe isotopic fractionation by gravitational separation inside porous planetesimals, by improving the model of Ozima and Nakazawa (1980) and Igarashi and Ozima (1988).

\section{Gravitational Separation Inside Porous Planetesimals with Size Distribution}

\section{Method of calculation}

When the size distribution of planetesimals is ex-

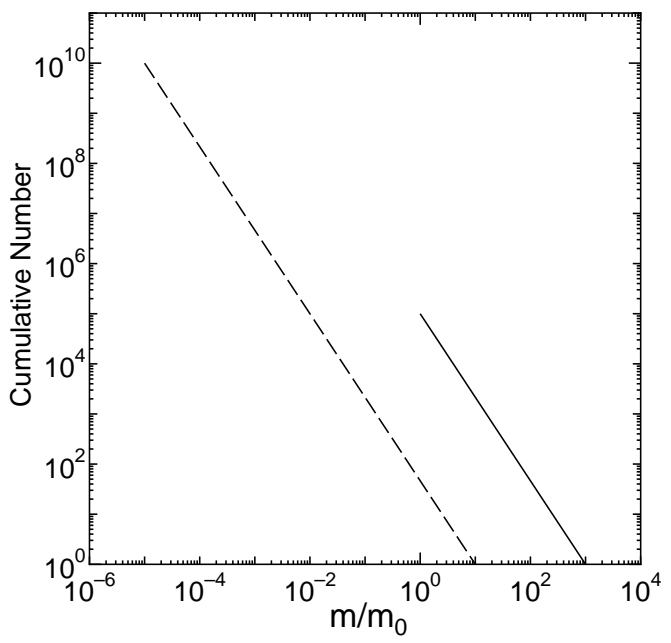

Fig. 2. An illustration of the cumulative number distribution for the size distribution given by Eq. (5) with a fixed total mass, which shows the relationship between the minimum and maximum masses $\left(m_{0}\right.$ and $\left.m_{\max }\right)$. Note that the cumulative number in this case is proportional to $m^{-5 / 3}$. The case with $N_{0}=10^{5}$ and the initial mass $m_{0}$ is shown by the solid line, where $m_{\max }=$ $10^{3} m_{0}$; $N_{0}$ is the initial number of planetesimals, which is approximated by the number of planetesimals with mass $m_{0}$ in this illustration, as most of the mass is in the low-mass end and the decrease of the number of planetesimals with mass $m_{0}$ can be neglected. For comparison, the case with the initial mass $m_{0}{ }^{\prime}=10^{-5} m_{0}$ is shown by the dashed line, where $N_{0}{ }^{\prime}=10^{5} N_{0}$ and $m_{\max }{ }^{\prime}=10 m_{0}$.

pressed with $N(R)\left(R_{0} \leq R \leq R_{\max }\right)$, the enrichment factor of a noble gas species $i$ in a protoplanet formed by accreting these planetesimals can be calculated as (Igarashi and Ozima, 1988)

$$
\bar{\alpha}_{i} \equiv \frac{\int_{R_{0}}^{R_{\max }} \alpha_{i}(R) N(R) R^{3} d R}{\int_{R_{0}}^{R_{\max }} N(R) R^{3} d R} .
$$

Isotopic fractionation is expressed in terms of $\delta$-values relative to the solar isotopic composition. In the case of $\mathrm{Xe}, \delta$ is defined as

$$
\delta_{i} \equiv\left({ }^{i} \mathrm{Xe} /{ }^{130} \mathrm{Xe}\right) /\left({ }^{i} \mathrm{Xe} /{ }^{130} \mathrm{Xe}\right)_{\text {solar }}-1
$$

Using $\bar{\alpha}_{i}$ defined by Eq. (7), $\delta_{i}$ can be written as (Igarashi and Ozima, 1988)

$$
\delta_{i}=\bar{\alpha}_{i} / \bar{\alpha}_{130}-1 .
$$

When the size distribution of planetesimals is given 


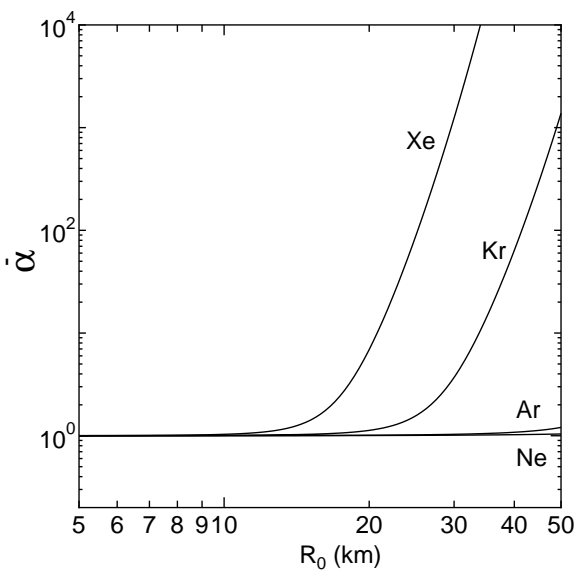

Fig. 3. Enrichment factor $\bar{\alpha}$ (Eq. (7)) averaged over the planetesimal size distribution, as a function of $R_{0}$.

by Eq. (5) or (6), the values of $m_{\max }$ can be approximately given by $m_{\max } \sim N_{0}{ }^{3 / 5} m_{0} \sim M_{\text {tot }}{ }^{3 / 5} m_{0}{ }^{2 / 5}$, where $M_{\text {tot }}$ and $N_{0}$ are the total mass and the initial total number of planetesimals with mass $m_{0}$, respectively (Fig. 2). This relation is consistent with the numerical results of Wetherill and Stewart $(1989,1993)$. Since $M_{\text {tot }}$ is conserved in the course of planetesimal accumulation, the above relation provides a relation between $m_{0}$ and $m_{\max }$ given as $m_{\max } / m_{0} \sim\left(M_{\text {tot }} / m_{0}\right)^{3 / 5}$. If we assume that $M_{\text {tot }}=$ $M_{\oplus}$ and the material density of a planetesimal is given by $\rho_{s}=3 \mathrm{~g} \mathrm{~cm}^{-3}$, we can rewrite this relation into the relation between $R_{0}$ and $R_{\max }$ given by

$$
R_{\max } / R_{0} \simeq 200 \times\left(\frac{R_{0}}{1 \mathrm{~km}}\right)^{-3 / 5}
$$

In the above, we adopt $3 \mathrm{~g} \mathrm{~cm}^{-3}$ as the material density instead of $2 \mathrm{~g} \mathrm{~cm}^{-3}$ used in Ozima and Nakazawa (1980), as this value is often used in recent numerical simulation of planetary accretion (e.g., Wetherill and Stewart, 1993). The main results of the present work are not affected by the choice of this value.

The initial size of a planetesimal formed as a result of gravitational instability of the dust layer in the solar nebula is estimated to be several $\mathrm{km}$, but significant uncertainty can be expected in the actual initial size of planetesimals. Therefore, we consider a rather wide range of values of $R_{0}(5-50 \mathrm{~km})$, and corresponding values of $R_{\max }$ are calculated from the relation (10).

\section{Elemental fractionation}

Figure 3 shows the plots of $\bar{\alpha}_{i}$ defined by Eq. (7) as a function of $R_{0}\left(R_{\max }\right.$ is calculated by Eq. (10)) for four noble gas species. When $R_{0}$ is smaller than about $10 \mathrm{~km}$, no significant enrichment is seen for all the four species.

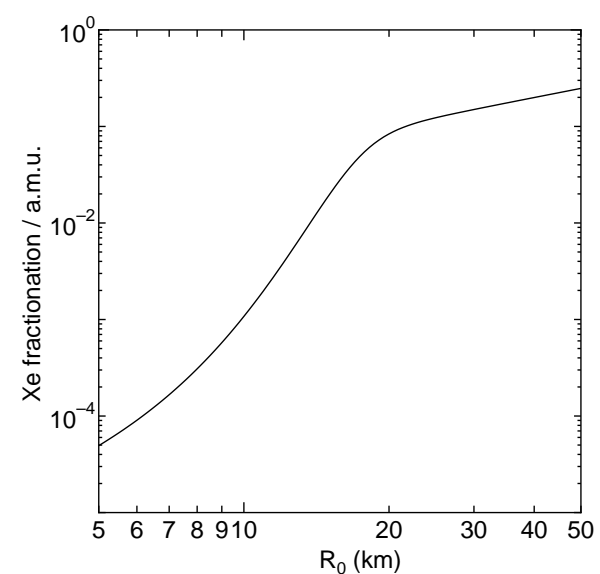

Fig. 4. Xe isotopic fractionation per a.m.u. as a function of $R_{0}$.

The enrichment of Xe becomes significant when $R_{0} \gtrsim 10$ $\mathrm{km}$. In this case, $R_{\max } \simeq 500 \mathrm{~km}$ (Eq. (10)), and a considerable number of planetesimals are larger than the critical size for Xe defined by Eq. (3). As a result, the enrichment of Xe becomes notable for $R_{0} \geqslant 10 \mathrm{~km}$, while other lighter gases are less affected because their critical sizes are larger. We find that the enrichment of Xe becomes comparable to the observed value $\left(\sim 3 \times 10^{3}\right.$; Igarashi and Ozima, 1988; Ozima and Podosek, 1983) when $R_{0} \simeq 30$ $\mathrm{km}\left(R_{\max } \simeq 800 \mathrm{~km}\right)$, although $\bar{\alpha}_{i}$ depends quite sensitively on $R_{0}$ (Fig. 3). For this value of $R_{0}$, the enrichment factor of $\mathrm{Kr}$ is $\sim 6$, which is against the observation, while the enrichment of $\mathrm{Ar}$ and $\mathrm{Ne}$ is negligible.

\section{Isotopic fractionation}

Figure 4 shows the plots of Xe fractionation per a.m.u. as a function of $R_{0}$. We find rapid increase of the degree of fractionation with increasing $R_{0}$, although we note the change in the functional form, which reflects the different $A \mu$-dependence of $\alpha$ (Fig. 1) for small and large bodies (i.e., $A \mu \lesssim 1$ and $A \mu>>1$ ). When approximated by a linear fractionation, the observed value of Xe fractionation per a.m.u. is $\simeq 0.04$ (Ozima and Podosek, 1983). We find that the calculated value becomes comparable to the observed one when $R_{0} \simeq 16-17 \mathrm{~km}$, although again a quite sensitive dependence on $R_{0}$ is seen.

Isotopic fractionation lines calculated for various values of $R_{0}$ are shown in Fig. 5. As we have mentioned above, the isotopic fractionation pattern is similar to the observed fractionation of terrestrial Xe when $R_{0} \simeq 16-$ $17 \mathrm{~km}$, except for ${ }^{129} \mathrm{Xe}$, whose excess can be attributed to the contribution of the decay of ${ }^{129} \mathrm{I}$ (Ozima and Podosek, 1983). However, as the dependence of the slope on $R_{0}$ is so strong, changing the value of $R_{0}$ by several $\mathrm{km}$ results in completely different fractionation patterns (Fig. 5). 


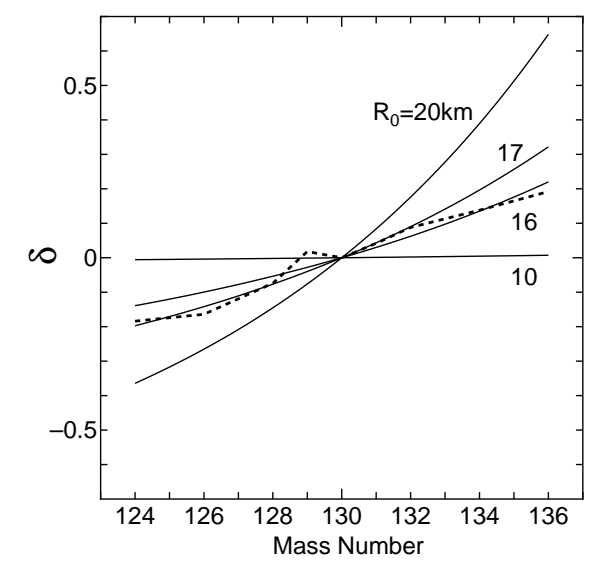

Fig. 5. Isotopic fractionation lines of Xe for various values of $R_{0}$. The isotopic fractionation pattern of Xe in the Earth's atmosphere relative to the surface-correlated Xe in a lunar mare soil (which is regarded to be close to the solar Xe composition) is also shown by the dashed line, after Ozima and Podosek (1983).

The results for the enrichment factor of Xe requires that $R_{0} \simeq 30 \mathrm{~km}$ (Fig. 3), while those for its isotopic fractionation suggests $R_{0} \simeq 16-17 \mathrm{~km}$, and in both cases a slight change in the value of $R_{0}$ produces completely different results. Therefore, it seems difficult to produce the result that can explain both the elemental abundance and the isotopic fractionation of terrestrial $\mathrm{Xe}$ with the simple model considered in the above calculations.

In the above calculations, we assumed that a protoplanet (proto-Earth) accretes planetesimals with the size distribution given by Eq. (6). Exactly speaking, we have to multiply Eq. (6) with the collision rate between the protoplanet and a planetesimal with a given size, as the collision rate also depends on the size of a planetesimal through the weak size-dependence of the random velocity of planetesimals. If we assume that the random velocity is large enough to neglect the effect of the solar gravity during encounter and that the random velocity is determined by a balance between the gas drag from the solar nebula and gravitational scattering by the protoplanet (e.g., Wetherill and Stewart, 1993), we can show that the collision rate is proportional to $m^{-1 / 15}$ or $R^{-1 / 5}$, thus the value of $q$ in Eq. (6) becomes 5.8, rather than 6. However, the mass-dependence of the random velocity and collision rate changes in different velocity regimes (e.g., Wetherill and Stewart, 1993; Ohtsuki et al., 2002). Taking these into account, we also calculated the enrichment and isotopic fractionation of Xe for various values of $q$ in the range of $5.6 \leq q \leq 6.4$ (Sano, 2000). Although a smaller value of $q$ results in a relatively large number of large planetesimals (Eq. (6)) and thus facilitates the gravitational capture of noble gases, the results we found were similar to the above case with $q=6$. The enrichment of $\mathrm{Xe}$ becomes comparable to the terrestrial value when 30 $\mathrm{km} \leq R_{0} \lesssim 35 \mathrm{~km}$, and its isotopic fractionation becomes close to the observed value when $15 \mathrm{~km} \lesssim R_{0} \lesssim 20 \mathrm{~km}$; again, the dependence on $R_{0}$ was quite sensitive.

\section{DISCUSSION}

In the present work, we investigated the fractionation of terrestrial Xe by gravitational separation inside porous planetesimals, taking account of the size distribution of planetesimals consistent with recent theoretical studies of planetary accretion. We find that the ranges of the parameter (i.e., the values of $R_{0}$ ) that would account for the isotopic fractionation and the total amount of terrestrial Xe separately, but the dependence on $R_{0}$ was very strong and the two parameter ranges were found not to overlap. For the value of $R_{0}$ that results in the observed enrichment of $\mathrm{Xe}$, significant enrichment of $\mathrm{Kr}$ was also found, in contrast to the observation. The derived values of $R_{0}$ $(\sim 15 \mathrm{~km}$ or $\sim 30 \mathrm{~km})$ were quite large compared to the initial size of planetesimals ( several $\mathrm{km}$ ) inferred from the theory of the gravitational instability of the dust layer in the solar nebula (Goldreich and Ward, 1973). We assumed the gravitational equilibrium for the distribution of noble gases inside planetesimals (Ozima and Nakazawa, 1980; Igarashi and Ozima, 1988), but the time needed for diffusion to occur can be too long to verify this assumption, especially for smaller planetesimals (Zahnle et al., 1990a). Furthermore, the gravitational separataion model has a difficulty that it alone cannot reproduce the observed abundance and isotopic composition of lighter noble gases in the terrestrial atmosphere. These results suggest that the fractionation of terrestrial Xe cannot be explained if we only consider the gravitational separation inside porous planetesimals with a size distribution, although it has been sometimes regarded as a promising mechanism (e.g., Ozima and Igarashi, 1989; Igarashi, 1995).

The other category of models for fractionating noble gas isotopes assumes vigorous hydrodynamic escape of a primordial atmosphere from Earth (e.g., Hunten et al., 1987; Sasaki and Nakazawa, 1988; Zahnle et al., 1990b; Pepin, 1991). Both gravitational separation and hydrodynamic models require the fractionated gases must be mixed with a source having low $\mathrm{Xe} / \mathrm{Kr}$ ratio and unfractionated isotopic ratios (Dauphas, 2003). The mantle is assumed to be such a source in some models (e.g., Pepin, 1991), while others consider the contribution of an external component delivered by icy planetesimals, such as comets (e.g., Owen et al., 1992; Dauphas, 2003). The latter model depends on the experimentally observed depletion of Xe relative to $\mathrm{Kr}$ when trapped in amorphous water ice, and also on the assumption that the noble gas 
isotopic composition of comets is solar. However, the dynamical history of comets and their noble gas composition are still unknown. The icy materials could have been delivered to the terrestrial planets in various ways, such as gas drag (e.g., Adachi et al., 1976) or radial diffusion due to gravitational scattering between planetesimals and protoplanets (e.g., Ohtsuki and Tanaka, 2003) during the early stage of the solar system evolution, and also as comets from the Uranus-Neptune region and the Kuiper Belt in the later stage (e.g., Morbidelli et al., 2000). Therefore, not only better understanding of the formation processes of the terrestrial planets, but also further understanding of the dynamical history and the composition of small icy bodies in the outer solar system will be necessary to clarify the origin and evolution of the atmospheres of terrestrial planets.

Acknowledgments-We thank Dr. Kazuo Saito for continuous encouragement and comments on the manuscript. We also thank Dr. Naoyoshi Iwata for discussion and encouragement. This work was supported by NASA's Origins of Solar Systems Program through grant NAG5-13043 to K.O.

\section{REFERENCES}

Adachi, I., Hayashi, C. and Nakazawa, K. (1976) The gas drag effect on the elliptical motion of a solid body in the primordial solar nebula. Prog. Theor. Phys. 56, 1756-1771.

Dauphas, N. (2003) The dual origin of the terrestrial atmosphere. Icarus 165, 326-339.

Goldreich, P. and Ward, W. R. (1973) The formation of planetesimals. Astrophys. J. 183, 1051-1061.

Hunten, D. M., Pepin, R. O. and Walker, J. C. G. (1987) Mass fractionation in hydrodynamic escape. Icarus $\mathbf{6 9}, 532-549$.

Igarashi, G. (1995) Primitive xenon in the Earth. Volatiles in the Earth and Solar System (Farley, K. A., ed.), 70-80, AIP Conf. Proc. 341.

Igarashi, G. and Ozima, M. (1988) Origin of isotopic fractionation of terrestrial xenon. Proc. NIPR Symp. Antarct. Meteorites 1, 315-320.

Kortenkamp, S. J., Kokubo, E. and Weidenschilling, S. J. (2000) Formation of planetary embryos. Origin of the Earth and Moon (Canup, R. M. and Righter, K., eds.), 85-100, Univ. of Arizona Press.

Morbidelli, A., Chambers, J., Lunine, J. I., Petit, J. M., Robert, F., Valsecchi, G. B. and Cyr, K. E. (2000) Source regions and timescales for the delivery of water to the Earth. Meteorit. Planet. Sci. 35, 1309-1320.

Nakagawa, Y., Hayashi, C. and Nakazawa, K. (1983) Accumulation of planetesimals in the solar nebula. Icarus $\mathbf{5 4}, 361-$
376.

Ohtsuki, K. and Tanaka, H. (2003) Radial diffusion rate of planetesimals due to gravitational encounters. Icarus $\mathbf{1 6 2}$, 47-58.

Ohtsuki, K., Stewart, G. R. and Ida, S. (2002) Evolution of planetesimal velocities based on three-body orbital integrations and growth of protoplanets. Icarus 155, 436-453.

Owen, T., Bar-Nun, A. and Kleinfeld, I. (1992) Possible cometary origin of heavy noble gases in the atmospheres of Venus, Earth, and Mars. Nature 358, 43-46.

Ozima, M. and Igarashi, G. (1989) Terrestrial noble gases: Constraints and implications on atmospheric evolution. Origin and Evolution of Planetary and Satellite Atmospheres (Atreya, S. K., Pollack, J. B. and Matthews, M. S., eds.), 306-327, Univ. of Arizona Press.

Ozima, M. and Nakazawa, K. (1980) Origin of rare gases in the Earth. Nature 284, 313-316.

Ozima, M. and Podosek, F. A. (1983) Noble Gas Geochemistry. Cambridge University Press, 367 pp.

Ozima, M. and Podosek, F. A. (2002) Noble Gas Geochemistry. 2nd ed., Cambridge University Press, 286 pp.

Pepin, R. O. (1991) On the origin and early evolution of terrestrial planet atmospheres and meteoritic volatiles. Icarus $\mathbf{9 2}$, 2-79.

Sano, M. (2000) Origin of the Earth's atmosphere by gravitational separation inside planetesimals: Effect of planetesimal size distribution based on numerical simulation of planetesimal accumulation. Bachelor Thesis, Yamagata University (in Japanese).

Sasaki, S. and Nakazawa, K. (1988) Origin of isotopic fractionation of terrestrial Xe: Hydrodynamic fractionation during escape of the primordial $\mathrm{H}_{2}-\mathrm{He}$ atmosphere. Earth Planet. Sci. Lett. 89, 323-334.

Takahashi, A. (1999) Origin of rare gases in the Earth's atmosphere: Gravitational separation inside planetesimals. Bachelor Thesis, Yamagata University (in Japanese).

Tolstikhin, I. N. and O'Nions, R. K. (1994) The earth's missing xenon: A combination of early degassing and of rare gass loss from the atmosphere. Chem. Geol. 115, 1-6.

Wetherill, G. W. and Stewart, G. R. (1989) Accumulation of a swarm of small planetesimals. Icarus 77, 330-367.

Wetherill, G. W. and Stewart, G. R. (1993) Formation of planetary embryos: Effects of fragmentation, low relative velocity, and independent variation of eccentricity and inclination. Icarus 106, 190-204.

Zahnle, K., Pollack, J. B. and Kasting, J. F. (1990a) Xenon fractionation in porous planetesimals. Geochim. Cosmochim. Acta 54, 2577-2586.

Zahnle, K., Kasting, J. F. and Pollack, J. B. (1990b) Mass fractionation of noble gases in diffusion-limited hydrodynamic hydrogen escape. Icarus 84, 502-527. 